\title{
SPHECIDAE Y CRABRONIDAE (HYMENOPTERA) DE ALGUNOS MUNICIPIOS DEL CENTRO Y SUR DE TAMAULIPAS, MÉXICO
}

\section{Jorge Víctor HorTa Vega ${ }^{1}$, Oswaldo Noel Pinson DomíngueZ², Ludivina Barrientos Lozano ${ }^{1}$ \& Alfonso Correa SANDOVal ${ }^{1}$}

1División de Estudios de Posgrado e Investigación, Instituto Tecnológico de Cd. Victoria. Blvd. Emilio Portes Gil 1301 Pte. Victoria, Tamps. 87010, MÉXICO.

jhortavega@yahoo.com.mx; ludivinab@yahoo.com; agutierr@uat.edu.mx 2Unidad Multidisciplinaria Agronomía y Ciencias de la Universidad Autónoma de Tamaulipas. Campus Victoria, Tamps. 87149, MÉXICO.

oswaldonoel@yahoo.com

\section{RESUMEN}

Se registran 124 especies y 44 géneros de avispas esfécidas de localidades de once municipios de Tamaulipas, México. Veintitrés especies y tres géneros son nuevos registros para el país y 94 especies y 26 géneros son nuevos registros para el Estado de Tamaulipas. Crabronidae y Sphecidae comprenden 98 y 26 especies, respectivamente. Las especies más abundantes son Ammophila picipes y Trypoxylon (Trypargilum) spinosum. La especie Ammophila picipes también es la de mayor distribución. Con la información disponible, para la República Mexicana se conocen 103 géneros y 658 especies y para Tamaulipas 54 géneros y 142 especies.

Palabras Clave: Avispas solitarias, Sphecidae, Crabronidae, Tamaulipas.

\begin{abstract}
One hundred and twenty four species and forty four genera of sphecoid wasps collected at localities of eleven municipalities of Tamaulipas, Mexico were recorded. Twenty three species and three genera are new records for the country meanwhile 94 species and 26 genera are new records for the state of Tamaulipas. Crabronidae and Sphecidae comprise 98 and 26 species respectively. The most abundant species were Ammophila picipes and Trypoxylon (Trypargilum) spinosum. Ammophila picipes is the most widely distributed species. From the 103 genera and 658 species recorded in Mexico 54 genera (52\%) and 142 species $(21.6 \%)$ have been found in Tamaulipas.
\end{abstract}

Key Words: Solitary wasps, Sphecidae, Crabronidae, Tamaulipas.

\section{INTRODUCCIÓN}

Las avispas incluidas dentro de la familia Sphecidae por Bohart \& Menke (1976) son especies no sociales que se describen como solitarias o depredadoras por cazar 
individualmente insectos o arañas para el aprovisionamiento de sus nidos con presas paralizadas (O'Neill 2001). Estos himenópteros han sido objeto de múltiples y amplios estudios, entre otros, ecológicos (Evans \& O'Neill 1988), farmacológicos (Piek \& Spanjer 1986), etológicos (Tinbergen 1958, 1972) y agronómicos (Lai 1988; Funasaki et al. 1988; Menke 1992).

Debido a la gran diversidad en formas, tamaño, comportamiento y ecología, la sistemática de estos insectos varía de acuerdo a los diferentes autores. En la clásica revisión mundial del grupo por Bohart \& Menke (1976) se clasifican como familia Sphecidae con once subfamilias. En estudios posteriores, las subfamilias se reagrupan y se designan como familias dentro de la superfamilia Apoidea. Por un lado, Finnamore \& Michener (1993) hacen una catalogación de nueve familias agrupadas como serie Spheciformes, y por otro lado, Melo (1999) propone sólo cuatro familias, que junto con Apidae, las ubica todas como superfamilia Apoidea. Las cuatro familias de esfeciformes son: Heterogynaidae, Ampulicidae, Sphecidae y Crabronidae.

Las avispas esfeciformes son cosmopolitas con unas 8,000 especies alrededor del mundo (Finnamore \& Michener 1993), de las que cerca de 1,200 especies se presentan en la región Neártica (Finnamore 1993), además de endemismos, las diseminadas por el hombre y las que presentan una amplia tolerancia climática (Bohart \& Menke 1976). En México, de acuerdo a una amplia revisión bibliográfica reciente (Ruíz et al. 2002), se tiene registro de 615 especies en 91 géneros.

Los estudios sobre esfeciformes en México son escasos por falta de especialistas. Algunas instituciones contienen en sus colecciones entomológicas ejemplares de estos grupos de insectos pero ninguna mantiene un continuo seguimiento sobre la catalogación sistemática y de estudios de su distribución. En algunos trabajos sobre Hymenoptera realizados en el noreste del país se incluyen a los esfécidos (Aquino \& Ruíz 1990; Varela et al. 1998) sin embargo, al no ser uno de los grupos de principal atención, sus colectas no han sido intensas. No obstante recientemente fue publicada una extensa revisión de especies de esfeciformes registradas para la República Mexicana (Ruíz et al. 2002).

El país forma parte de dos grandes regiones biogeográficas: la Neotropical y la Neártica. La región Neotropical alcanza sus límites septentrionales en las planicies neolonesas y tamaulipecas por el este y en las planicies sonorenses por el oeste; en cuanto a la región Neártica, sus límites meridionales se localizan en el centro de la República Mexicana (Ruíz \& Coronado 2002; Morrone 2001). De esta manera, al converger componentes de ambas regiones, se espera que el Estado de Tamaulipas sea mucho más rico en biodiversidad de lo que la literatura muestra, lo cual muy probablemente se refleje en la fauna de esfeciformes.

Este trabajo, motivado en sus orígenes por los componentes neuroactivos de los venenos de artrópodos, pretende ampliar el conocimiento de avispas depredadoras y 
contribuir con los estudios de biodiversidad de esta región del país donde se entrelazan diversos ecosistemas.

\section{MATERIALES Y MÉTODOS}

\section{Colecta de especímenes y localidades}

La colecta de especímenes se realizó manualmente con red entomológica y estacionariamente con trampas Malaise. Los ejemplares fueron preservados en alcohol al 70\% y refrigerados hasta su montaje con técnicas entomológicas estándar. Las colectas se realizaron de Febrero de 1999 a Julio de 2002.

Las localidades de colecta (15) presentaron altitudes desde el nivel del mar hasta los 1,150 msnm, y se distribuyeron en once municipios del centro y sur de Tamaulipas (Fig. 1). Los métodos de captura y sus intensidades no fueron los mismos en todas las localidades. Sin embargo, se cubrieron distintos tipos de hábitat en la región: matorral espinoso, matorral inerme y subinerme, selva baja caducifolia, bosque de pino-encino, agricultura de temporal, vegetación riparia, selva mediana subperennifolia, selva baja espinosa, vegetación halófila y bosque mesófilo de montaña (INEGI 1981; Treviño-Carreón \& Valiente-Banuet 2005). Cuando la colecta fue manual, en cada localidad se exploró un área de aproximadamente una hectárea.

\section{Determinación taxonómica}

En la determinación a género y especie de los ejemplares se utilizaron claves especializadas (Bohart 1993, Bohart 1994, Bohart 1996a,1996b, Bohart 2000, Bohart \& Grissell 1975, Bohart \& Kimsey 1979, Bohart \& Menke 1963, Bohart \& Menke 1976, Bohart \& Schlinger 1957, Coville 1982, Evans \& Matthews 1968, Hensen 1988, Krombein \& Shanks-Gingras 1984, Leclercq 1991, Malloch \& Rower 1930, Menke 1996, Menke \& Fernández 1996, Parker 1962, 1969, Pate 1948, Sandhouse 1940, Scullen 1972, van Lith 1975, 1979) y se realizaron visitas a las colecciones entomológicas de la Academia de Ciencias de California en San Francisco, CA., del Museo de Entomología "Bohart" de la Universidad de California en Davis, CA. y de la Unidad Académica Multidisciplinaria Agronomía y Ciencias de la U.A.T., Cd. Victoria, Tamps. Asimismo, algunos de los ejemplares se enviaron al "Instituto de Investigaciones Ammophila", Beesbe, AZ., en donde fueron determinados por el Dr. Arnold S. Menke.

\section{Análisis de datos}

Para establecer diferencias en la longitud de los ejemplares en relación a los métodos de colecta se realizaron comparaciones de dos medias independientes utilizando la prueba $t$ con una $p$ de 0.05 . La diversidad de especies por localidades se evaluó con el índice de Shannon-Wiener (Odum 2000). 
Horta et al.: Sphecidae y Crabronidae (Hymenoptera) de Tamaulipas, México

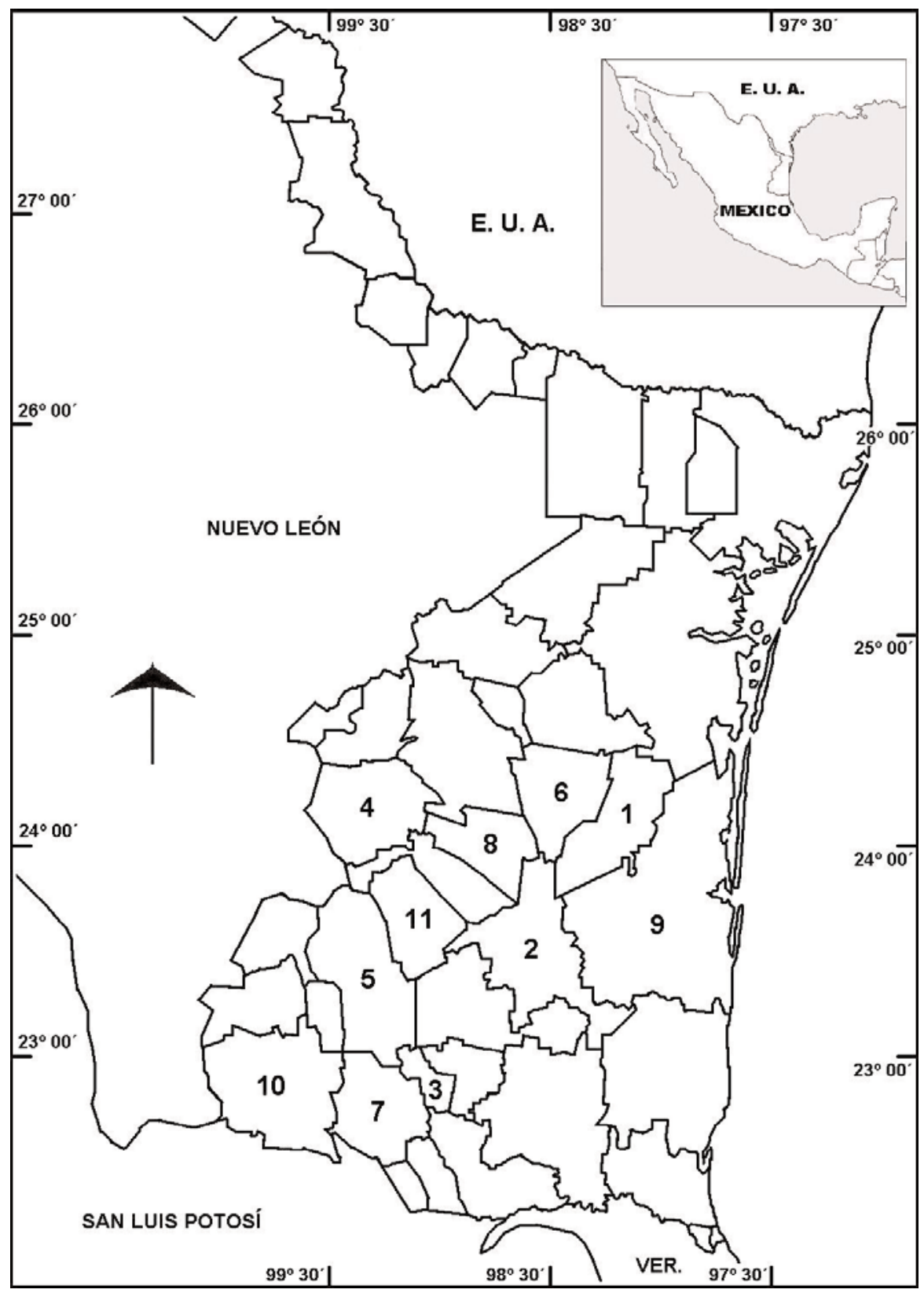

Figura 1

Municipios de Tamaulipas en donde se colectaron ejemplares. 1) Abasolo, 2) Casas, 3) Gómez Farías, 4) Hidalgo, 5) Jaumave, 6) Jiménez, 7) Ocampo, 8) Padilla, 9) Soto la Marina, 10) Tula, 11) Victoria. 


\section{RESULTADOS}

\section{Abundancia y diversidad}

Se colectaron en total 1,206 ejemplares (Apéndice). Se determinaron 124 especies, incluyendo en dos de ellas dos distintas subespecies y 22 morfoespecies en donde no se logró la identificación específica. De la familia Crabronidae se colectaron 629 ejemplares mientras que de Sphecidae fueron 577. La familia con mayor riqueza de especies fue Crabronidae con 98, en tanto que de Sphecidae se colectaron 26 especies.

Las especies más abundantes fueron Ammophila picipes Cameron, 1888 con 176 ejemplares y Trypoxylon (Trypargilum) spinosum Cameron, 1889 con 105 individuos colectados. Ammophila picipes fue la especie que presentó mayor distribución, colectándose en 12 localidades. Trypoxylon (Trypargilum) spinosum junto con Ammophila centralis Cameron, 1888 ocuparon el segundo sitio en cuanto a la amplitud de distribución al presentarse en siete de las 15 localidades (ver Apéndice). Treinta y dos especies están representadas por un solo ejemplar y 54 del total se colectaron en alguna de las localidades (ver Apéndice).

En la figura 2 se presenta el incremento de especies en relación al número de ejemplares colectados. Los intervalos de clase fueron de 100 ejemplares cada uno, excepto el último que incluyó a 106. Se observa claramente una tendencia de incremento continuo prácticamente a todo lo largo del estudio, indicando que el número de especies en esta región de México podría ser mayor a lo encontrado aquí. Por lo tanto es necesario realizar un mayor esfuerzo de colecta para obtener un registro de especies más completo.

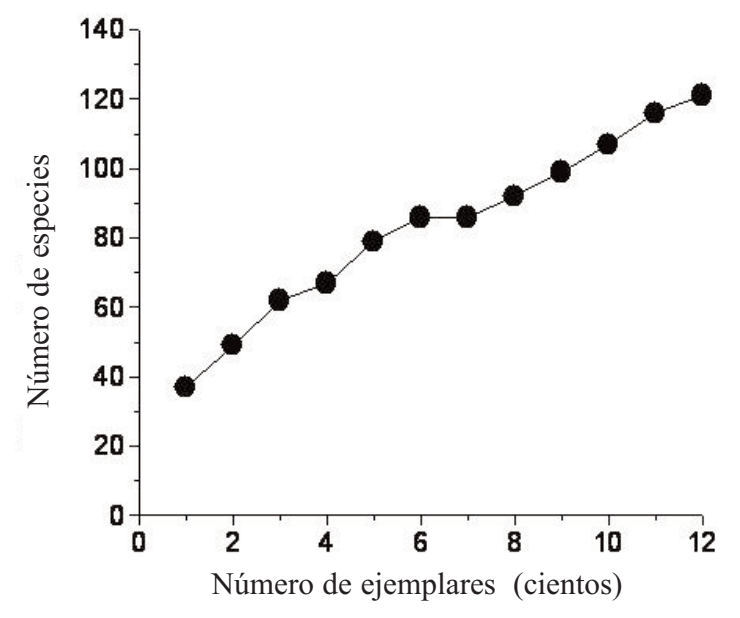

Figura 2

Acumulación de especies en función del número de ejemplares colectados. 


\section{Localidades más colectadas}

La localidad con mayor número de ejemplares colectados fue el Cañón del Novillo (ver Apéndice) en donde se capturaron 291 ejemplares; la siguieron Cd. Victoria (255), Los Cedros (188) y Alta Cima (144). Las especies presentes en cada una de ellas fueron 60, 38, 43 y 37, respectivamente. De estas localidades el Cañón del Novillo presentó la mayor diversidad con un valor de índice de Shannon de 3.50.

\section{Métodos de colecta}

De los 1,206 ejemplares, 786 se capturaron con red entomológica y 420 en trampa Malaise. El 53\% fueron hembras, es decir, sin tendencia de algún sexo en relación con el tipo de captura. Sin embargo, sí se encontraron diferencias en cuanto al tamaño de los ejemplares. Las avispas capturadas con red presentaron una longitud promedio de $17.4 \mathrm{~mm}$, la cual fue significativamente mayor que los $11.8 \mathrm{~mm}$ de longitud promedio de las avispas atrapadas con la trampa.

En las especies también se observaron diferencias; exclusivamente con red se capturó el $36.4 \%$ de las especies en este estudio, exclusivamente colectadas con trampa Malaise fueron el $31.4 \%$ y el resto de las especies (32.2\%) indistintamente por ambos métodos.

\section{DISCUSIÓN}

\section{Sphecidae y Crabronidae en México}

De las 124 especies colectadas, 23 de ellas no se citan en Ruíz et al. (2002) para México. En el caso de los 44 géneros, cinco de ellos (Argogorytes, Metanysson, Miscophus, Moniaecera y Stenogorytes) no se mencionan en dicha revisión. Dos familias, Crabronidae y Sphecidae, de las cuatro familias distribuidas a nivel mundial (Melo 1999) están representadas en este estudio. La familia Crabronidae contribuyó con 19 nuevos registros para México y cuatro en el caso de Sphecidae. Estos resultados concuerdan con los datos de Ruíz et al. (2002) de la familia Crabronidae como la de mayor diversidad de esfeciformes en México.

En el estudio de R. M. Bohart (2000) sobre la tribu Gorytini de la Región Neotropical, el cual no se incluye en Ruíz et al. (2002), se presentan 21 especies para México en nueve géneros. Uno de esos géneros y nueve de esas especies se describen como nuevas para la ciencia. Los géneros Argogorytes Ashmead, 1899, representado por una nueva especie ( $A$. mexicalis Bohart, 2000), y Stenogorytes Schrottky, 1911, asignado como nuevo estatus, son de los nueve descritos para la República Mexicana (Bohart 2000). De esta manera, el presente estudio contribuye con tres nuevos registros de géneros para México (Metanysson, Miscophus y Moniaecera). Asimismo, algunos de los ejemplares colectados aquí y aún no determinados específicamente podrían ser nuevas especies como es el caso de ejemplares del género Ammophila, de acuerdo a Menke, especialista del grupo. 


\section{Sphecidae y Crabronidae en Tamaulipas}

En el listado de especies de Ruíz et al. (2002) se citan 23 especies de 10 géneros para el Estado de Tamaulipas. Bohart (2000) de la tribu Gorytini describe dos especies de dos géneros colectadas en Tamaulipas. Una de ellas nueva especie para la ciencia (Clitemnestra irwini Bohart, 2000) y capturada también en los estados de Chiapas, Guerrero, Campeche y Morelos. De estudios regionales se conocen tres publicaciones (Aquino \& Ruíz 1990; Ruíz \& Coronado 1998; Varela et al. 1998) que describen en conjunto 21 géneros para el estado. En el trabajo de Varela et al. (1998) se incluye al género Sagenista Bohart, 1967, que no se menciona en el listado de Ruíz et al. (2002) pero si en Bohart (2000) (en donde se describe la nueva especie Sagenista pilosa Bohart 2000) pero con material que no fue colectado en el Estado de Tamaulipas. En este trabajo 26 géneros y 94 especies son nuevos registros para el estado. Asimismo, en el material colectado no se encontraron 18 especies y 10 géneros ya antes conocidos de los anteriores estudios y revisiones, lo cual puede deberse entre otros factores, a los métodos de colecta empleados, su intensidad y/o a las condiciones ecológicas prevalecientes durante el trabajo de campo.

Las especies nuevas de la tribu Gorytini (Bohart 2000), las posibles nuevas especies entre algunos ejemplares de este estudio, los numerosos nuevos registros de especies y de géneros, así como el constante ascenso en el número de especies reclutadas durante el período de estudio, conforman fuertes evidencias de la gran diversidad por descubrir de esfeciformes en México y en particular en el Estado de Tamaulipas. Por lo tanto, se requiere de más estudios para seguir contribuyendo con el conocimiento de este interesante grupo de insectos. Esto es particularmente importante en localidades como el Cañón del Novillo del Mpio. de Victoria, que con 60 especies colectadas, resultó ser la de mayor diversidad en esta investigación y es considerada área susceptible de protección por las autoridades gubernamentales.

\section{Métodos de colecta}

Del total de especies, el 36.4\% y el 31.4\%, fueron capturadas exclusivamente con red y trampa Malaise, respectivamente, lo que sugiere que ambos métodos se complementan para conocer la composición taxonómica de estos grupos de insectos. Estos resultados son semejantes a los encontrados por Horta et al. (2003) sobre géneros de esfeciformes. Es difícil determinar que factores y con que intensidad influyen en el tipo y número de especies colectadas con cada uno de los métodos. La captura manual con red permite la exploración de una mayor variedad de ambientes o tipos de vegetación en combinación con la variada conducta entre las distintas especies, y la fácil visualización de avispas grandes es un factor adicional. Con trampa Malaise se favorece la colecta de especies con similares hábitos en la búsqueda de alimento, presas y en el cortejo, pero tiene la ventaja de una mayor y continua ventana de tiempo para la captura. 
Horta et al.: Sphecidae y Crabronidae (Hymenoptera) de Tamaulipas, México

\section{AGRADECIMIENTOS}

Agradecemos al Consejo Nacional de Ciencia y Tecnología (CONACYT) y a Fondos Mixtos CONACYT-GOBIERNO DE TAMAULIPAS por el financiamiento que hizo posible esta publicación. Agradecemos al Dr. Wojciech J. Pulawski y Dra. Helen K. Court de la Academia de Ciencias de California en San Francisco y la Dra. Lynn S Kimsey y Dr. Steve Heydon del Museo Bohart de la University of California, Davis, CA., por su apoyo durante las visitas a sus colecciones entomológicas. Al Dr. Arnold S. Menke por la determinación de ejemplares y valiosas sugerencias. Al Dr. Enrique Ruíz Cancino por sus comentarios y la aportación de algunos ejemplares. A la M.C. Regina María Brussolo Ceballos por la revisión final del manuscrito. A la Sra. Ofelia Crespo Ortiz y a la Srita. Marcela E. Macías Pérez por su colaboración secretarial.

\section{LITERATURA CITADA}

Aquino, S.M. \& E. Ruíz C. 1990. Géneros de Vespidae, Sphecidae y Pompilidae (Hymenoptera) en el sur de Tamaulipas. Biotam 2 (2): 47-54.

Bohart, R.M. 1993. A synopsis of Central American and Caribbean Oxybelus (Hymenoptera, Sphecidae). Insecta Mundi 7 (3): 159-168.

. 1994. A key to the genus Tachytes in America North of Mexico with descriptions of three new species (Hymenoptera, Sphecidae, Larrinae). Proceedings of the Entomological Society of Washington 96 (2): 342-349.

- 1996a. A review of Bembicinus (Hymenoptera: Sphecidae: Stizini) in North and Central America. Proceedings of the Entomological Society of Washington 98 (3): 517-526.

. 1996b. A review of the genus Bicyrtes (Hymenoptera: Sphecidae, Nyssoninae, Bembicini). Insecta Mundi 10 (1-4): 139-152.

.2000. A review of Gorytini in the Neotropical Region (Hymenoptera: Sphecidae: Bembicinae). Contributions on Entomology, International 4 (2): 111-259.

Bohart, R.M. \& A.S. Menke. 1963. A reclassification of the Sphecinae. with a revision of the nearctic species of the tribes Sceliphronini and Sphecini (Hymenoptera, Sphecidae). University of California Publications in Entomology 30 (2): 91-182.

. 1976. Sphecid wasps of the world. a generic revision. University of California Press. Berkeley. 695 pp.

Bohart, R.M. \& E.E. Grissell. 1975. California wasps of the subfamily Philanthinae (Hymenoptera: Sphecidae). Bulletin of the California Insect Survey $19.92 \mathrm{pp}$.

Bohart, R.M. \& E.I. Schlinger. 1957. California wasps of the genus Oxybelus (Hymenoptera: Sphecidae, Crabroninae). Bulletin of the California Insect Survey 4 (4): 103-134.

Bohart, R.M. \& L.S. Kimsey. 1979. A key to the species of Ectemnius in America North of Mexico with notes and description of a new species (Hymenoptera: Sphecidae). Proceedings of the Entomological Society of Washington 81(3): 486-498.

Coville, R.E. 1982. Wasps of the genus Trypoxylon subgenus Trypargilum in North America (Hymenoptera: Sphecidae). University of California Publications in Entomology 97, 147 pp. 
Evans, H.E. \& K.M. O'Neill. 1988. The natural history and behavior of North American beewolves. Cornell University Press. Ithaca, NY. 278 pp.

Evans, H.E. \& R.W. Matthews. 1968. North american Bembix, a revised key and suggested grouping. Annals of the Entomological Society of America 61 (5): 1284-1299.

Finnamore, A.T. 1993. Series Spheciformes. pp. 280-306. In: H. Goulet and J. T. Huber (Eds.). Hymenoptera of the World: An Identification Guide to Families. Research Branch Agriculture Canada. Ottawa, Canada.

Finnamore, A.T. \& C.D. Michener. 1993. Superfamily Apoidea pp. 279-357. In: H. Goulet and J. T. Huber (eds.). Hymenoptera of the World: An Identification Guide to Families. Research Branch Agriculture Canada. Ottawa, Canada.

Funasaki, G.Y., P. Lai, L.M. Nakahara, J.W. Beardsley \& A.K. Ota. 1988. A review of biological control introductions in Hawai: 1890-1985. Procedings of the Hawaii Entomological Society 28:105-160.

Hensen, R.V. 1988. Revision of the nominate subgenus Chalybion Dahlbom (Hymenoptera, Sphecidae). Tijdschrift voor Entomologie 131: 13-64.

Horta, V.J.V., M. Vanoye E., O. Pinson D. \& E. Ruíz C. 2003. Géneros de Sphecoidea (Hymenoptera) del Centro de Tamaulipas, México. Biotam, n.s. 14(3):47-62.

INEGI. 1981. Síntesis Geográfica del Estado de Tamaulipas. Secretaría de Programación y Presupuesto. 157 pp.

Krombein K.V. \& S. Shanks-Gingras. 1984. Revision of North American Liris Fabricius (Hymenoptera: Sphecoidea: Larridae). Smithsonian Contributions to Zoology 404, 96 pp.

Lai, P.Y. 1988. Biological control: a positive point of view. Procedings of the Hawaii Entomological Society 28:179-190.

Leclercq, J. 1991. Hymenopteres sphecides crabroniens du genre Ectemnius Dahlbom trouves en Amerique Latine. Bulletin de la Société Royale des Sciences de Liége 1: 3-29.

Malloch, J.R. \& S.A. Rower. 1930. New forms of sphecoid wasps of the genus Didineis Wesmael. Proceedings of the United States National Museum 77 (14): 1-7.

Melo, G.A.R. 1999. Phylogenetic relationships and classification of the major lineages of Apoidea (Hymenoptera), with emphasis on crabronid wasps. Scientific Papers. Natural History Museum, The University of Kansas 14:1-55.

Menke, A.S. 1992. Mole cricket hunters of the genus Larra in the New World (Hymenoptera: Sphecidae, Larrinae). Journal of Hymenoptera Research 1 (1): 175-234. . 1996. The Ammophilini of Costa Rica; an identification guide (Hymenoptera: Sphecidae: Sphecinae). Journal of Hymenoptera Research 5: 190-202.

Menke, A.S. \& F. Fernández C. 1996. Claves ilustradas para las subfamilias, tribus y géneros de esfécidos neotropicales (Apoidea: Sphecidae). Revista de Biología Tropical 44 (Supplement 2): 1-68.

Morrone, J.J. 2001. Biogeografia de América Latina y el Caribe. Manuales \& Tesis Sea. Vol. 3. Cyted, Orcyt-Unesco \& Sea (eds.). 150 Pp.

Odum, E.P. 2000. Ecology. Editorial McGrawHill-Interamericana, España. 639 pp.

O'Neill, K.M. 2001. Solitary wasps: Behavior and Natural History. Cornell University Press. Ithaca, NY, 406 pp. 
Parker, F.D. 1962. On the subfamily Astatinae, with a systematic study of the genus Astata of America North of Mexico (Hymenoptera: Sphecidae). Annals of the Entomological Society of America 55 (6): 643-659.

. 1969. On the subfamily Astatinae. Part VI. The American species in the genus Dryudella Spinola (Hymenoptera: Sphecidae). Annals of the Entomological Society of America 62 (5): 963-976.

Pate, V.S.L. 1948. A review of the genus Moniaecera (Hymenoptera: Sphecidae: Pemphilidini). Transactions of the American Entomological Society. 74: 41-60.

Piek, T. \& W. Spanjer. 1986. Chemistry and pharmacology of solitary wasp venoms. pp. 161-307. In: T. Piek (ed.), Venoms of the Hymenoptera: biochemical, pharmacological and behavioural aspects. Academic Press, New York.

Ruíz, C.E. \& J.M. Coronado B. 1998. Sphecidae (Hymenoptera) en la reserva de la biosfera "El Cielo". In: Memorias del $10^{\circ}$ Encuentro de Investigación Científica y Tecnológica del Golfo de México. Reynosa, Tamaulipas. p. 16. . 2002. Artrópodos terrestres de los estados de Tamaulipas y Nuevo León, México. Serie Publicaciones Científicas, Cidaff-Uat, No. 4. Cd. Victoria, Tamaulipas, México.

Ruíz, C.E., J.M. Coronado B., S.E. Varela \& J.V. Horta Vega. 2002. Sphecoidea. pp. 657670. In: J. Llorente-Bousquets y J. Morrone (eds.). Biodiversidad, taxonomía y biogeografía de artrópodos de México: hacia una síntesis de su conocimiento Vol III. Facultad de Ciencias, UNAM, México, D. F.

Sandhouse, G.A. 1940. A review of the neartic wasp of the genus Trypoxylon (Hymenoptera: Sphecidae). The American Midland Naturalist 24: 133-174.

Scullen, H.A. 1972. Review of the genus Cerceris Latreille in Mexico and Central America (Hymenoptera: Sphecidae). Smithsonian Contributions to Zoology 110, 121 pp.

Tinbergen, N. 1958. Naturalistas Curiosos. Salvat Editores, España. 260 pp. - 1972. The animal in its world, explorations of ethologist 1932-1972: Field Studies. Harvard University Press, Cambridge, Mass. 296 pp.

Treviño-Carreón, J. \& A. Valiente-Banuet. 2005. La vegetación de Tamaulipas y sus principales asociaciones vegetales. pp. 22-46. In: L. Barrientos Lozano, A. Correa Sandoval, J. V. Horta Vega y J. García Jiménez (eds.). Biodiversidad Tamaulipeca Vol. 1. Instituto Tecnológico de Cd. Victoria, DGEST-SEP, Cd. Victoria, Tamps., México.

van Lith, J. P. 1975. Neotropical species of Psen and Pseneo (Hymenoptera, Sphecidae, Psenini). Tijdschrift voor Entomologie 118 (1): 1-41.

. 1979. The new world genus Pluto (Hymenoptera, Sphecidae, Psenini). Tijdschrift voor Entomologie 122 (6): 127-239.

Varela, S. E., E. Ruíz C., \& L. M. Pérez. 1998. Himenópteros en localidades de Gómez Farías y Ocampo, Tamaulipas, México. Biotam 10 (2): 47-58. 


\section{APÉNDICE}

Listado de especies de Crabronidae y Sphecidae, ordenadas alfabéticamente, número de ejemplares colectados, localidades y número de ejemplares por localidad.

\begin{tabular}{|c|c|c|}
\hline FAMILIA/ESPECIE & Ejemplares & $\begin{array}{c}\text { Localidades } \\
\text { (No. de ejemplares) }\end{array}$ \\
\hline \multicolumn{3}{|l|}{ CRABRONIDAE } \\
\hline Argogorytes sapellonis Baker, 1907 & 1 & $\mathrm{LC}(1)$ \\
\hline Astata mexicana Cresson, 1881 & 1 & $\mathrm{CN}(1)$ \\
\hline Astata occidentalis Cresson, 1881 & 2 & $\mathrm{LT}(2)$ \\
\hline Astata unicolor Say, 1824 & 3 & $\mathrm{CN}(2), \mathrm{LC}(1)$ \\
\hline Bembicinus mexicanus Handlirsch, 1892 & 12 & $\mathrm{CN}(2), \mathrm{CV}(9), \mathrm{SJ}(1)$ \\
\hline Bembix multipicta F. Smith, 1873 & 15 & $\mathrm{CN}(15)$ \\
\hline Bicyrtes fodiens Handlirsch, 1889 & 3 & $\operatorname{LCD}(3)$ \\
\hline Bicyrtes variegata Oliver, 1789 & 1 & LP(1) \\
\hline Bicyrtes viduata Handlirsch, 1889 & 23 & $\mathrm{CN}(3), \mathrm{CV}(15), \mathrm{LC}(1), \mathrm{LN}(1), \mathrm{LCD}(3)$ \\
\hline Bothynostethus sp. & 1 & $\operatorname{CV}(1)$ \\
\hline Cerceris californica californica Cresson, 1865 & 1 & $\mathrm{CN}(1)$ \\
\hline Cerceris conifrons Mickel, 1916 & 2 & $\mathrm{LT}(1), \mathrm{RP}(1)$ \\
\hline Cerceris cribosa Spinola, 1841 & 2 & $\mathrm{LC}(1), \mathrm{SJ}(1)$ \\
\hline Cerceris dilatata dilatata Spinola, 1841 & 12 & $\mathrm{CN}(5), \mathrm{CV}(3), \mathrm{SJ}(4)$ \\
\hline Cerceris femurrubrum Viereck y Cockerell, 1904 & 2 & $\mathrm{CN}(2)$ \\
\hline Cerceris finitima Cresson, 1865 & 3 & $\mathrm{CN}(2), \mathrm{CV}(1)$ \\
\hline Cerceris insolita chiriquensis Cameron, 1890 & 1 & $\mathrm{LC}(1)$ \\
\hline Cerceris kennicottii kennicottii Cresson, 1865 & 3 & $\mathrm{CN}(1), \mathrm{CV}(1), \mathrm{LT}(1)$ \\
\hline Cerceris kennicottii zapoteca Saussure, 1867 & 4 & $\mathrm{CN}(1), \mathrm{LC}(1), \mathrm{LT}(1), \mathrm{SJ}(1)$ \\
\hline Cerceris parkeri Scullen, 1972 & 3 & $\mathrm{CN}(2), \mathrm{CV}(1)$ \\
\hline Cerceris rufinoda Cresson, 1865 & 3 & LT(3) \\
\hline Cerceris truncata Cameron, 1890 & 3 & $\mathrm{CV}(2), \mathrm{LCD}(1)$ \\
\hline Didineis texana Cresson, 1873 & 1 & $\mathrm{AC}(1)$ \\
\hline Dryudella caerulea Cresson, 1881 & 32 & CV(1), LC(1), LT(29), LN(1) \\
\hline Ectemnius excavatus W. Fox, 1892 & 2 & $\mathrm{LC}(2)$ \\
\hline Larropsis sp. & 1 & $\mathrm{LT}(1)$ \\
\hline Lestica producticollis Packard, 1866 & 2 & $\mathrm{LC}(1), \mathrm{AC}(1)$ \\
\hline Lestica sp. & 1 & $\mathrm{AC}(1)$ \\
\hline Liris argentatus Palisot de Beauvois, 1811 & 11 & $\mathrm{CN}(3), \mathrm{CV}(1), \mathrm{LC}(1), \mathrm{AC}(2), \mathrm{TU}(4)$ \\
\hline Liris beatus Cameron, 1889 & 4 & $\mathrm{CN}(2), \mathrm{LC}(1), \mathrm{AC}(1)$ \\
\hline Liris liparus Krombein and Shanks Gingras, 1984 & 6 & $\mathrm{CN}(1), \mathrm{AC}(5)$ \\
\hline Liris mescalero mescalero Pate, 1943 & 3 & $\mathrm{AC}(1), \mathrm{LT}(1), \mathrm{TU}(1)$ \\
\hline Liris mexicanus Krombein and Shanks Gingras, 1984 & 11 & $\mathrm{CN}(6), \mathrm{CV}(2), \mathrm{LC}(1), \mathrm{AC}(1), \mathrm{SJ}(1)$ \\
\hline Liris muspa Pate, 1943 & 3 & $\mathrm{LC}(1), \mathrm{AC}(2)$ \\
\hline Liris nearcticus Krombein and Shanks Gingras, 1984 & 8 & $\mathrm{CN}(7), \mathrm{LT}(1)$ \\
\hline Liris panamensis panamensis Cameron 1889 & 7 & $\mathrm{CN}(2), \mathrm{LC}(3), \mathrm{AC}(2)$ \\
\hline Liris partitus Krombein and Shanks Gingras, 1984 & 1 & $\mathrm{AC}(1)$ \\
\hline Liris sp. & 15 & $\mathrm{CN}(9), \mathrm{CV}(1), \mathrm{AC}(2), \mathrm{LT}(3)$ \\
\hline
\end{tabular}


Horta et al.: Sphecidae y Crabronidae (Hymenoptera) de Tamaulipas, México

\begin{tabular}{|c|c|c|}
\hline FAMILIA/ESPECIE & Ejemplares & $\begin{array}{c}\text { Localidades } \\
\text { (No. de ejemplares) }\end{array}$ \\
\hline Liris vincenti Krombein and Shanks Gingras, 1984 & 3 & $\mathrm{CN}(1), \mathrm{CV}(1), \mathrm{AC}(1)$ \\
\hline Megistommum splendidum Handlirsch, 1888 & 2 & $\mathrm{LC}(2)$ \\
\hline Metanysson solani Cockerell, 1895 & 1 & $\mathrm{LBC}(1)$ \\
\hline Microbembex monodonta Say , 1824 & 6 & $\mathrm{LN}(5), \operatorname{LCD}(1)$ \\
\hline Miscophus sp. & 1 & $\mathrm{LT}(1)$ \\
\hline Moniaecera abdominalis W. Fox, 1895 & 3 & $\mathrm{CN}(2), \mathrm{LCD}(1)$ \\
\hline Oxybelus argenteopilosus Cameron, 1891 & 1 & $\mathrm{LT}(1)$ \\
\hline Philanthus gibbosus Fabricius, 1775 & 1 & $\operatorname{LL}(1)$ \\
\hline Pison sp. & 2 & $\mathrm{LC}(2)$ \\
\hline Pluto albifacies Malloch, 1933 & 2 & $\mathrm{LT}(2)$ \\
\hline Pluto spangleri van Lith, 1979 & 6 & $\mathrm{CN}(2), \mathrm{LT}(4)$ \\
\hline Pluto suffusus W. Fox, 1898 & 9 & $\mathrm{CN}(7), \mathrm{CV}(2)$ \\
\hline Pseneo montezuma Cameron, 1891 & 3 & $\mathrm{LC}(1), \mathrm{AC}(2)$ \\
\hline Psenulus aztecus Bohart \& Grissell, 1969 & 2 & $\mathrm{AC}(2)$ \\
\hline Psenulus sp. & 1 & $\mathrm{AC}(1)$ \\
\hline Rhopalum sp. & 2 & $\mathrm{LC}(2)$ \\
\hline Solierella plenoculoides W. Fox, 1893 & 7 & $\mathrm{CN}(7)$ \\
\hline Sphecius convallis Patton, 1879 & 1 & $\mathrm{~EB}(1)$ \\
\hline Sphecius grandis Say, 1823 & 1 & TU(1) \\
\hline Sphecius speciosus Drury, 1773 & 1 & $\mathrm{LP}(1)$ \\
\hline Stenogorytes notabilis Handlirsch, 1888 & 4 & $\mathrm{LC}(3), \mathrm{OC}(1)$ \\
\hline Tachysphex sp. 1 & 13 & $\mathrm{CN}(2), \mathrm{CV}(1), \mathrm{AC}(2), \mathrm{LT}(7), \mathrm{RP}(1)$ \\
\hline Tachysphex sp. 2 & 2 & $\mathrm{CV}(2)$ \\
\hline Tachysphex sp. 3 & 1 & $\mathrm{AC}(1)$ \\
\hline Tachysphex sp. 4 & 10 & $\mathrm{CN}(10)$ \\
\hline Tachysphex sp. 5 & 7 & $\mathrm{CN}(7)$ \\
\hline Tachysphex sp. 6 & 1 & $\mathrm{CN}(1)$ \\
\hline Tachysphex sp. 7 & 15 & $\mathrm{CN}(8), \mathrm{LC}(1), \mathrm{LT}(5), \mathrm{OC}(1)$ \\
\hline Tachytes abdominalis Say, 1823 & 3 & $\mathrm{CN}(1), \mathrm{LT}(1), \mathrm{LL}(1)$ \\
\hline Tachytes amazonus F. Smith, 1856 & 1 & $\mathrm{CN}(1)$ \\
\hline Tachytes chrysocercus Rohwer, 1811 & 13 & $\mathrm{CN}(9), \mathrm{CV}(1), \mathrm{LT}(1), \mathrm{LCD}(1), \mathrm{RP}(1)$ \\
\hline Tachytes chrysopyga obscurus Cresson, 1873 & 1 & $\mathrm{CN}(1)$ \\
\hline Tachytes distinctus F. Smith, 1856 & 2 & $\operatorname{LT}(1), \operatorname{LCD}(1)$ \\
\hline Tachytes floridanus Rohwer, 1920 & 1 & $\mathrm{CV}(1)$ \\
\hline Tachytes harpax Patton, 1880 & 1 & $\mathrm{LC}(1)$ \\
\hline Tachytes intermedius Viereck, 1906 & 1 & $\operatorname{LL}(1)$ \\
\hline Tachytes obductus W. Fox, 1892 & 1 & $\mathrm{CV}(1)$ \\
\hline Tachytes pennsylvanicus Banks, 1921 & 7 & $\mathrm{CN}(1), \mathrm{CV}(1), \mathrm{LC}(2), \mathrm{LT}(2), \mathrm{RP}(1)$ \\
\hline Trachypus gracilis Cameron, 1890 & 2 & $\mathrm{CN}(2)$ \\
\hline Trachypus mexicanus Saussure, 1867 & 21 & $\mathrm{CN}(3), \mathrm{CV}(1), \mathrm{LT}(2), \mathrm{SJ}(15)$ \\
\hline Trypoxylon (Trypargilum) carinifrons Cameron, 1889 & 1 & $\mathrm{AC}(1)$ \\
\hline Trypoxylon (Trypargilum) lactitarse Saussure, 1867 & 14 & $\mathrm{CN}(2), \mathrm{CV}(1), \mathrm{LC}(10), \mathrm{AC}(1)$ \\
\hline Trypoxylon (Trypargilum) mexicanum Saussure, 1867 & 23 & $\mathrm{LC}(22), \mathrm{SJ}(1)$ \\
\hline
\end{tabular}




\begin{tabular}{|c|c|c|}
\hline FAMILIA/ESPECIE & Ejemplare & $\begin{array}{c}\text { Localidades } \\
\text { (No. de ejemplares) }\end{array}$ \\
\hline \multicolumn{2}{|l|}{ Trypoxylon (Trypargilum) nitidum aztecum Saussure, 186716} & $\mathrm{CN}(2), \mathrm{CV}(6), \mathrm{LC}(5), \mathrm{AC}(2), \mathrm{LN}(1)$ \\
\hline Trypoxylon (Trypargilum) pectorale Richards, 1934 & 1 & $\mathrm{CV}(1)$ \\
\hline Trypoxylon (Trypargilum) spinosum Cameron, 1889 & 105 & $\begin{array}{c}\mathrm{CN}(22), \mathrm{CV}(37), \mathrm{LC}(28), \mathrm{AC}(8), \\
\mathrm{LT}(4), \mathrm{LP}(3), \mathrm{TU}(3)\end{array}$ \\
\hline Trypoxylon (Trypargilum) superbum F. Smith, 1873 & 5 & $\mathrm{LC}(5)$ \\
\hline Trypoxylon (Trypargilum) tridentatum Packard, 1867 & 3 & LT(2), TU(1) \\
\hline Trypoxylon (Trypoxylon) carinatum Say, 1837 & 3 & $\mathrm{CN}(1), \mathrm{AC}(2)$ \\
\hline Trypoxylon (Trypoxylon) grupo figulus sp. 1 & 36 & $\mathrm{CN}(3), \mathrm{CV}(2), \mathrm{LC}(27), \mathrm{AC}(2), \mathrm{LT}(2)$ \\
\hline Trypoxylon (Trypoxylon) grupo figulus sp. 2 & 5 & $\mathrm{LC}(4), \mathrm{AC}(1)$ \\
\hline Trypoxylon (Trypoxylon) grupo figulus sp. 3 & 4 & $\mathrm{LC}(3), \mathrm{AC}(1)$ \\
\hline Trypoxylon (Trypoxylon) grupo figulus sp. 4 & 1 & $\mathrm{CV}(1)$ \\
\hline Trypoxylon (Trypoxylon) grupo figulus sp 3 & 4 & $\mathrm{LC}(3), \mathrm{AC}(1)$ \\
\hline Trypoxylon (Trypoxylon) grupo figulus sp 4 & 1 & CV $(1)$ \\
\hline Trypoxylon (Trypoxylon) grupo figulus sp 5 & 4 & $\mathrm{CN}(2), \mathrm{LC}(1), \mathrm{AC}(1)$ \\
\hline Trypoxylon (Trypoxylon) grupo figulus sp. 1 & 36 & $\mathrm{CN}(3), \mathrm{CV}(2), \mathrm{LC}(27), \mathrm{AC}(2), \mathrm{LT}(2)$ \\
\hline Trypoxylon (Trypoxylon) johnsoni W. Fox, 1891 & 4 & $\mathrm{CN}(2), \mathrm{CV}(2)$ \\
\hline Trypoxylon (Trypoxylon) punctivertex Richards, 1934 & 2 & $\mathrm{LC}(2)$ \\
\hline Zanysson texanus Cresson, 1872 & 6 & $\mathrm{CN}(1), \mathrm{CV}(1), \mathrm{AC}(1), \mathrm{LT}(1), \mathrm{LBC}(2)$ \\
\hline Astata mexicana Cresson, 1881 & 1 & $\mathrm{CN}(1)$ \\
\hline Argogorytes sapellonis Baker, 1907 & 1 & $\mathrm{LC}(1)$ \\
\hline Astata occidentalis Cresson, 1881 & 2 & $\mathrm{LT}(2)$ \\
\hline Subtotal & 629 & \\
\hline \multicolumn{3}{|l|}{ SPHECIDAE } \\
\hline Ammophila breviceps F. Smith, 1856 & 13 & $\begin{array}{c}\mathrm{CN}(1), \mathrm{CV}(1), \mathrm{LT}(1), \mathrm{LN}(4), \mathrm{TU}(5), \\
\mathrm{RP}(1)\end{array}$ \\
\hline Ammophila centralis Cameron, 1888 & 70 & $\begin{array}{c}\mathrm{CN}(17), \mathrm{CV}(22), \mathrm{LC}(6), \mathrm{AC}(11), \\
\mathrm{LT}(4), \mathrm{SJ}(1), \mathrm{EB}(9)\end{array}$ \\
\hline Ammophila cleopatra Menke, 1964 & 16 & $\mathrm{LN}(13), \mathrm{TU}(1), \mathrm{RP}(2)$ \\
\hline Ammophila picipes Cameron, 1888 & 176 & $\begin{array}{c}\mathrm{CN}(44), \mathrm{CV}(49), \operatorname{LC}(4), \operatorname{AC}(59), \mathrm{LT}(3) \\
\mathrm{LN}(2), \mathrm{SJ}(1), \operatorname{LP}(2), \operatorname{LCD}(7), \\
\mathrm{TU}(2), \operatorname{RP}(1), \mathrm{OC}(2)\end{array}$ \\
\hline Ammophila procera Dahlbom, 1843 & 22 & $\mathrm{CN}(5), \mathrm{CV}(16), \mathrm{LC}(1)$ \\
\hline Ammophila sp. & 10 & $\mathrm{CN}(1), \mathrm{CV}(5), \mathrm{LN}(1), \mathrm{LCD}(1), \mathrm{TU}(2)$ \\
\hline Chalybion zimmermanni aztecum Saussure, 1867 & 2 & $\mathrm{CV}(1), \mathrm{LN}(1)$ \\
\hline Chalybion zimmermanni zimmermanni Dahlbom, 1843 & 328 & $\begin{array}{c}\mathrm{CN}(6), \mathrm{LC}(16), \mathrm{AC}(3), \mathrm{LT}(1), \\
\mathrm{LN}(1), \mathrm{TU}(1)\end{array}$ \\
\hline Chlorion cyaneum Dahlbom, 1843 & 1 & $\mathrm{RP}(1)$ \\
\hline Eremnophila aureonotata Cameron, 1888 & 47 & $\mathrm{CN}(9), \mathrm{CV}(33), \mathrm{LC}(5)$ \\
\hline Eremnophila melanaria Dahlbom, 1843 & 6 & $\mathrm{SJ}(6)$ \\
\hline Isodontia apicalis F. Smith, 1856 & 26 & LT(1), LP(8), LCD(1), TU(1), LL(15) \\
\hline Isodontia elegans F. Smith, 1856 & 7 & $\mathrm{CN}(4), \mathrm{AC}(1), \mathrm{SJ}(2)$ \\
\hline
\end{tabular}


Horta et al.: Sphecidae y Crabronidae (Hymenoptera) de Tamaulipas, México

\begin{tabular}{|c|c|c|}
\hline FAMILIA/ESPECIE & Ejemplares & $\begin{array}{c}\text { Localidades } \\
\text { (No. de ejemplares) }\end{array}$ \\
\hline Isodontia fuscipennis Fabricius, 1804 & 3 & $\mathrm{LC}(3)$ \\
\hline Isodontia mexicana Saussure, 1867 & 18 & $\begin{array}{c}\mathrm{CN}(7), \mathrm{CV}(3), \operatorname{LC}(1), \operatorname{LT}(2), \\
\operatorname{LP}(1), \operatorname{LCD}(4)\end{array}$ \\
\hline Palmodes dimidiatus De Geer, 1773 & 1 & $\mathrm{LN}(1)$ \\
\hline Podalonia sp. & 2 & $\mathrm{LN}(2)$ \\
\hline Podium rufipes Fabricius, 1804 & 4 & $\mathrm{LC}(3), \mathrm{AC}(1)$ \\
\hline Prionyx canadensis Provancher, 1887 & 1 & $\mathrm{LT}(1)$ \\
\hline Prionyx parkeri Bohart \& Menke, 1963 & 64 & $\begin{array}{c}\mathrm{CN}(20), \mathrm{CV}(25), \mathrm{AC}(1), \mathrm{LT}(9), \\
\mathrm{LN}(8), \operatorname{LL}(1)\end{array}$ \\
\hline Prionyx thomae Fabricius, 1775 & 14 & $\mathrm{AC}(8), \mathrm{LT}(3), \mathrm{LL}(2), \mathrm{OC}(1)$ \\
\hline Sceliphron assimile Dahlbom, 1843 & 9 & $\mathrm{CN}(1), \mathrm{LC}(5), \mathrm{LP}(1), \mathrm{LCD}(1), \mathrm{OC}(1)$ \\
\hline Sceliphron caementarium Drury, 1773 & 4 & CN(1), LN(1), TU(2) \\
\hline Sphex (Fernaldina) lucae Saussure, 1867 & 24 & $\begin{array}{c}\mathrm{CN}(2), \mathrm{LT}(3), \mathrm{LN}(1), \mathrm{LP}(15), \\
\mathrm{LCD}(1), \mathrm{LL}(2)\end{array}$ \\
\hline Sphex ichneumoneus Linnaeus, 1758 & 7 & $\mathrm{LC}(4), \mathrm{SJ}(3)$ \\
\hline Sphex tepanecus Saussure, 1867 & 2 & $\mathrm{LT}(2)$ \\
\hline Subtotal & 577 & \\
\hline TOTAL & 1,206 & \\
\hline
\end{tabular}

LCD, La Cascada, Abasolo; LL, La Lajilla, Casas; AC, Alta Cima, Gómez Farías; LC, Los Cedros, Gómez Farías, SJ, San José, Gómez Farías; EB, Ejido Balconcillos, Hidalgo; LN, Los Nogales, Jaumave; LBC, Libertad Campesina, Jiménez; OC, Ocampo, Cabecera Municipal; RP, Río Purificación, Padilla; LP, La Pesca, Soto la Marina; TU, Tula, Cabecera Municipal; CN, Cañón del Novillo, Victoria; LT, Los Troncones, Victoria; CV, Cd. Victoria (periferia nororiente), Victoria. 